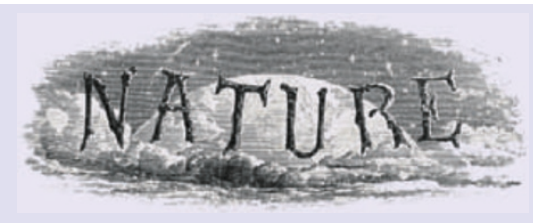

100 YEARS AGO

A Grass-snake which the writer had in his possession for eighteen months has just died. A fact which seems worthy of a note is the length of time during which this snake fasted. The last time the snake fed was June 11, 1904, the meal consisting of a small frog. From that time until the date of its death, February 2, it took no food, although constantly offered it. The animal thus existed for close on eight months without food. During the whole of this time it appeared in good health, and was, at times, most animated. No approach to hibernation was observed, and only for a little more than a week before its death did the snake seem out of health. The body was not unduly thin.

From Nature 23 February 1905.

\section{YEARS AGO}

The organization for the fishing and conservation of the Cœlacanths of the Comoro Islands created by the Institute de Recherche Scientifique de Madagascar... reports a new success: on November 12 last a further Latimeria was captured at Anjouan. This brings the total since 1938 to eight and is the finest yet, as regards both size and state of preservation, and by far the most interesting because it is the first near-adult female specimen which has come into our hands as well as the first of these precious fishes which anyone has been able to observe alive... Throughout the night - which the delighted population of Mutsamudu passed in singing and dancing to celebrate the capture - the Cœlacanth was watched over with admirable care by the chef de circonscription, taking turns with his adjoint, M. Solère. It seemed, although quite bewildered at the sequel to its ascent to the surface, to be taking the situation very well, swimming slowly by curious rotating movements of its pectoral fins, while the second dorsal and anal, likewise very mobile, served together with the tail as a rudder. After daybreak it became apparent that the light, and above all the sun itself, was upsetting the animal very much [and] the fish began to show more and more obvious signs of distress... At $14.45 \mathrm{hr}$. it was still swimming feebly; but at $\mathbf{1 5 . 3 0} \mathrm{hr}$. it had its belly in the air and only the fins and gill-covers were making agonized movements.

From Nature 26 February 1955. type. So Th-POK seems to be at the top of a gene-regulation hierarchy that controls T-cell fate.

Many tantalizing questions remain. What controls expression of the Th-POK gene in developing thymocytes? The observation that Th-POK synthesis is increased in thymocyte precursors that recognize class II MHC, but not class I MHC, implies that the gene's expression is regulated by engagement of class II MHC. This may be controlled by differences in T-cell receptor signalling on recognition of class I compared with class II MHC, as predicted by a popular model for CD4 versus CD8 lineage commitment (reviewed in refs 4,5). Further analysis of the regulation of Th-POK by T-cell-receptor signalling will be needed to test this idea.

What genes are regulated directly by Th-POK? The gene-expression programmes of CD4 and CD8 cells differ in terms of the production of GATA3, perforin (a T-cell effector protein), and CD4 and CD8 themselves. However, it is not yet clear whether these differences in gene expression are direct or indirect consequences of Th-POK activity.

Finally, self-reinforcing feedback loops are often used to 'lock in' cell-fate decisions, and there are hints that Th-POK is part of such a regulatory circuit. HD mice expressing class II MHC, but not class I MHC, show increased synthesis of messenger RNA for Th-POK in thymocyte precursor cells. However, the redirected CD8 T cells that ultimately develop in these mice lack Th-POK. Whether the requirement for Th-POK function to maintain Th-POK gene expression involves auto-regulation of the gene or involves other components of the gene regulatory hierarchy remains to be seen.

The quest to understand the CD4 versus CD8 lineage decision has focused mostly on the question of whether $\mathrm{MHC}$ recognition instructs the cell-fate decision (the instructive model), or whether cells choose their fate randomly and are then tested for the presence of the appropriate CD4 or CD8 molecule (the stochastic or selection model) $)^{9,10}$. These models, although useful, assumed that T-cell fate determination occurs as a single discrete step, but it has become clear that it is a multi-step process involving feedback and reinforcement. The identification of Th-POK as a key in the T-cell fate decision should open the way to deeper mechanistic insights into how cells are guided to their destiny.

Ellen A. Robey is in the Department of Molecular and Cell Biology, 471 Life Sciences Addition,

University of California, Berkeley, California 94720, USA.

e-mail:erobey@uclink.berkeley.edu

1. He, X. et al. Nature 433, 826-833 (2005).

2. Dave, V. P., Allman, D., Keefe, R., Hardy, R. R. \& Kappes, D. J.

Proc. Natl Acad. Sci. USA 95, 8187-8192 (1998).

3. Keefe, R., Dave, V., Allman, D., Wiest, D. \& Kappes, D. J. Science 286, 1149-1153 (1999)

4. Basson, M. A. \& Zamoyska, R. Immunol. Today 21, 509-514 (2000).

5. Germain, R. N. Nature Rev. Immunol. 2, 309-322 (2002).

6. Hernandez-Hoyos, G., Anderson, M. K., Wang, C., Rothenberg, E. V. \& Alberola-Ila, J. Immunity 19, 83-94 (2003).

7. Pai, S. Y. et al. Immunity $19,863-875$ (2003)

8. Taniuchi, I. et al. Cell 111, 621-633 (2002).

9. Janeway, C. A. Nature 335, 208-210 (1988).

10. Robey, E. A. et al. Cell 64, 99-107 (1991).

Planetary science

\title{
Being there
}

Kevin Zahnle

The protoplanets that collided to make the Earth may themselves have had atmospheres and oceans. Venus has vastly more argon and neon than Earth: fossil evidence, perhaps, of protoplanetary atmospheres?

$\mathrm{N}$ oble gases are the flotsam of the Solar System. They seem simple: they shun chemistry, they are difficult to freeze, and they accumulate in atmospheres. We see them as passive tracers of our cosmogonic theories, especially theories that address the origin and evolution of planetary volatiles. Perhaps it is a measure of our theories that every newly probed atmosphere has surprised us. Yet the temptation to read profound meaning into noble-gas abundances remains strong. On page 842 of this issue, Genda and Abe ${ }^{1}$ ask whether atmospheres can survive a series of giant impacts, such as the collision with Earth that formed our Moon. The answers are ambiguous, with argon agreeable but neon cryptic.
Current fashion posits four stages in the growth of an Earth: coagulation of grains into boulders; gathering of the boulders into aggregates of kilometre dimensions; runaway accretion of those aggregates into Moon-sized protoplanets; and giant collisions between the protoplanets to make planets ${ }^{2}$. The first three stages are thought to have taken no more than a million years in total, whereas the fourth played out over tens of millions of years or more.

Given how quickly stages 1-3 occurred, there is a fair likelihood that significant nebular gas was still present when the protoplanets emerged from runaway accretion. If the nebula was cold enough, and the protoplanets large enough, they would gravitationally capture significant primary atmospheres. 
When the nebula disappeared, the protoplanets would retain some of these atmospheres and thereby acquire noble gases from the solar nebula.

Genda and $A b e^{1}$ use a ruthlessly simplified model to describe how giant impacts interact with atmospheres ${ }^{3}$. They accept as a matter of course that much of the stricken hemisphere is lost, driven off by hydrodynamic jetting or the tangential momentum of a glancing blow. Their concern is with the hemisphere that is not hit directly.

Here the impact announces itself when the bow shock erupts through the surface. Indeed, the entire crust launches into space at high velocity, typically several kilometres per second. The crust drives a shock wave into the atmosphere that accelerates as it rises into thinner air. At some height the air reaches escape velocity, but escape is efficient only if the ground velocity approaches or exceeds the escape velocity ${ }^{3,4}$. Such high ground velocities are difficult to achieve if the planet itself survives; the highest ground motions achieved in successful Moon-forming impact simulations (a relatively gentle event) are typically less than half the escape velocity ${ }^{5}$. Genda and Abe therefore argue that most of a planet's atmosphere, including its primordial noble gases, is retained in giant impacts.

An ocean changes everything. When the crust hits the ocean, the ocean is driven outward at an even higher velocity and it is also vaporized. The resulting explosion of supercharged steam accelerates most of the overlying atmosphere to escape velocity and beyond (Fig. 2 in the supplementary information $\left.^{1}\right)$. Genda and Abe therefore argue that, with an ocean, most of the atmosphere (and most of the noble gas) is lost in giant impacts. Because they were there, the noble gases provide the best test of their hypothesis.

The authors take the minority view that bound water was common to all the building blocks of Earth and Venus, so that from the start the protoplanets each had their own oceans and atmospheres, and independent lives as infant Earths. A more popular view is that Earth and Venus became wet while still accreting, but only because they were struck by cold, wet protoplanets ejected from what is now the asteroid belt ${ }^{6}$; with modest revision, however, Genda and Abe's hypothesis could apply in this context as well.

To distinguish between Earth and Venus, Genda and Abe invoke the runaway greenhouse effect ${ }^{7}$. On worlds nearer the Sun, water evaporates into a thick atmosphere, whereas on more distant worlds it condenses as oceans and ice sheets. With oceans, the building blocks of Earth lost much more of their primary atmospheres than did the building blocks of Venus. In this way, Genda and Abe's model can account for Venus having 70 times more argon than Earth.

Of course there are caveats. Genda and Abe stress low-velocity collisions that merge the two protoplanets. A broader assessment of impact geometries and velocities suggests that most giant impacts are bounces, rather than mergers, in which both bodies emerge slightly smaller (and much hotter) amidst a spray of silicates ${ }^{5}$. In a high-speed bouncing impact between unequal planets, the smaller one could easily lose both its crust and its atmosphere.

But the big problem with gravitational capture of nebular noble gases is the abundance of neon. The neon:argon ratio on Venus, Earth, Mars and meteorites is invariably about $1 \%$ of what it is in the $\operatorname{Sun}^{8}$. Explaining this requires both a heroic theory of selective neon escape $e^{9}$ and some luck to make the neon:argon ratio always come out the same. It may work better to start from some other source of noble gases that discriminates against neon — say, extremely low-temperature condensates that quantitatively trap argon ${ }^{10}$ (neon freezes only if hydrogen freezes), or noble gas implantation by the solar wind into unaccreted grains and boulders ${ }^{11-13}$. If you merely wish to bury the argon, there is no limit to how much.
The new work offers a fresh look at planetary accretion. The argument that oceans speed the loss of atmospheres is more broadly applicable than the authors imply. It need not be restricted to atmospheres of solar composition acquired gravitationally; and it need not be restricted to water oceans or even liquid oceans. It applies generally during or before the giant-impact stage of accretion, and it could apply to satellites of the outer Solar System as easily as to Earth.

Kevin Zahnle is at the NASA Ames Research Center, Space Science Division, MS 245-3, Moffett Field,

California 94035-1000, USA.

e-mail:kevin.j.zahnle@nasa.gov

1. Genda, H. \& Abe, Y. Nature 433, 842-844 (2005).

2. Lissauer, J. J. Annu. Rev. Astron. Astrophys. 31, 129-174 (1993).

3. Chen, G. Q. \& Ahrens, T. J. Phys. Earth Planet. Int. 100, 21-26 (1997).

4. Genda, H. \& Abe, Y. Icarus 164, 149-162 (2003)

5. Agnor, C. \& Asphaug, E. Astrophys. J. Lett. 613, 157-160 (2004) 6. Morbidelli, A. et al. Meteorit. Planet. Sci. 35, 1309-1320 (2000).

7. Nakajima, S., Hayashi, Y. \& Abe, Y. J. Atmos. Sci. 49, 2256-2266 (1969)

8. Pepin, R. O. Icarus 92, 2-79 (1991).

9. Zahnle, K. J., Kasting, J. F. \& Pollack J. B. Icarus 84, 502-527 (1990)

10. Owen, T. et al. Nature 402, 269-270 (1999).

11. Wetherill, G. Icarus 46, 70-80 (1981).

12. Sasaki, S. Icarus 91, 29-38 (1991).

13. Ballentine, C., Marty, B., Lollar, B. S. \& Cassidy, M. Nature 433, 33-38 (2005).

Human immunodeficiency virus

\section{Refolding the envelope}

Peter D. Kwong

HIV has evolved to avoid neutralization by human antibodies. New atomic-level details reveal that such evasion involves substantial refolding of its exterior glycoprotein.

A defining feature of HIV is the ability to thrive for a decade or more despite sustained and vigorous immune attack. How does the virus remain able to infect host cells at the same time as evading immune surveillance? The solution resides largely in the molecular trickery of a single viral protein, gp120 - the component of the 'spikes' on the viral surface that binds to receptors on host cells.

Tantalizing new details of this trickery are revealed on page 834 of this issue ${ }^{1}$, where Chen and colleagues report the structure of the unliganded (receptor-free) form of gp120. Comparison with the previously determined structure $^{2}$ of gp120 in complex with one of its cell-surface receptors reveals that receptor binding induces roughly half of the protein to refold. Like a trick jigsaw puzzle that can form two entirely different pictures, the refolding preserves pieces of gp120's secondary structure, but reshuffles their location and relative orientation. In the viral spike, this reshuffling moves pieces of protein by more than $40 \AA$, rivalling the largest changes ever observed for a single protein domain.

Soon after HIV was identified as the aetiological agent of AIDS, it became clear that although infection prompts the human body to produce a large number of HIV-reactive antibodies, they are mostly ineffective at neutralizing the virus ${ }^{3}$. The machinery behind HIV's remarkable evasion involves a protective membrane around the virus and an ordered, two-receptor mechanism of entry into cells. The membrane is derived from a previous host cell and forms the outer surface of HIV - its envelope. The only viral proteins that protrude through this membrane are the envelope glycoproteins, gp 120 and gp41: three copies of each make up a viral spike.

The two-receptor mechanism involves the virus first binding to the CD4 glycoprotein on the surface of a potential host cell. This binding induces changes in gp120 that permit it to interact with a second cellsurface receptor, or co-receptor ${ }^{4,5}$. This mechanism is not essential for viral propagation, 